\title{
Corticosteroids for Multiple Sclerosis: I. Application for Treating Exacerbations
}

\author{
Elliot M. Frohman, ${ }^{\dagger \dagger}$ Anjali Shah, ${ }^{* \star}$ Eric Eggenberger, ${ }^{\S}$ Luanne Metz, ${ }^{\text {II }}$ Robert Zivadinov, ${ }^{\|}$and \\ Olaf Stüve
}

\begin{abstract}
Departments of $*$ Neurology, ${ }^{\dagger}$ Ophthalmology, and ${ }^{\ddagger}$ Physical Medicine and Rehabilitation, University of Texas Southwestern Medical Center at Dallas, Dallas, Texas 75235; Department of ${ }^{\S}$ Neurology and Ophthalmology, Michigan State University, East

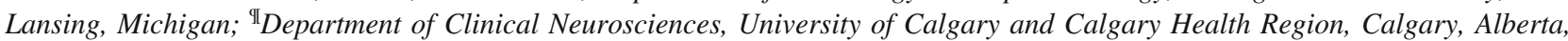
Canada; Department of "Neurology, Buffalo Neuroimaging Analysis Center, The Jacobs Neurological Institute, State University of New York at Buffalo, Buffalo, New York; and ${ }^{\#}$ Neurology Section, Medical Service, Veterans Affairs North Texas Health Care System, Dallas, Texas.
\end{abstract}

Summary: Multiple sclerosis (MS) is an inflammatory demyelinating disorder characterized by a multiphasic course of neurological exacerbations, periods of clinical remission, and, in most patients, ultimately progressive deterioration of functional capabilities. The relapsing-remitting phase of the disease involves acute interruption in neurological functioning relating to areas of inflammation in discrete central-tract systems. The treatment of MS exacerbations with anti-inflammatory agents such as corticosteroids and adrenocorticotropic hormone has represented an established practice throughout the neurology community. Although there is scientific rationale supporting application of these agents for this purpose, the broad diversity of approaches to using these drugs in clinical practice is a derivative of expert opinion and anecdotal experience. Ultimately, the treatment of MS-related exacerbations is part science, but mostly art. This review discusses the pharmacology of these agents, to better understand how they may act to mitigate attacks and to provide some practical formulations for how to use them in the clinic for the benefit of patients. Key Words: Corticosteroids, ACTH, pulse steroids.

\section{DEFINING AN EXACERBATION}

Exacerbations of multiple sclerosis (MS) are defined by episodes of neurological dysfunction that occur spontaneously and are not on the basis of an alternative etiology. Bona fide exacerbations are typically expected to last for at least 24 hours, and often involve the evolution of novel symptoms, not previously experienced by the patient. In some cases, however, the reemergence of old symptoms that persist can also be considered a true exacerbation, particularly when not explained on the basis of some other etiology, such as infection, stress, or elevation of core body temperature (i.e., the Uhthoff phenomenon). ${ }^{1}$ Symptoms that fluctuate in severity in a given individual according to their body temperature and with physical or emotional stress would not be consid-

Address correspondence and reprint requests to: Elliot M. Frohman, M.D., Ph.D., Department of Neurology, University of Texas Southwestern Medical Center at Dallas, 5323 Harry Hines Blvd., Dallas, TX 75235. E-mail: elliot.frohman@utsouthwestern.edu. ered a relapse unless the worsening is significantly greater in severity or duration than the range of their fluctuations. An important caveat to this definition is that infectious processes are now well known to trigger signaling pathways (e.g., the Toll-like receptor transduction sequence) that could culminate in the production of a proinflammatory context, resulting in production of an infection-associated exacerbation of MS. ${ }^{2}$ Recognition of this mechanism has led to reevaluation of what constitutes true exacerbations (as opposed to pseudoexacerbations), and has evident implications for the treatment and safety of patients.

The treatment of MS-related exacerbations has traditionally involved the use of anti-inflammatory treatments such as corticosteroids (CS) and adrenocorticotropic hormone (ACTH). ${ }^{3}$ The principal objectives for the application of these treatment strategies have been to accelerate recovery from the neurologic deficits sustained from the new area of inflammatory demyelination, as well as to mitigate the severity of the attack and reduce potentially persistent residual deficits. 
To understand how these agents serve to exert such activities on MS exacerbations, it is useful to review some of the established actions that are attributable to CS and ACTH.

\section{ACTION OF CORTICOSTEROIDS}

Corticosteroids are very potent effectors in the prevention and suppression of inflammation caused by chemical, immunological, infectious, and mechanical stressors. Because of their anti-inflammatory and immunosuppressive properties, these agents have been used for decades as standard therapy for numerous inflammatory disorders, including include asthma, systemic lupus erythematosus, vasculitis, inflammatory bowel disease, MS, myasthenia gravis, and rheumatoid arthritis. The effect of $\mathrm{CS}$ on the immune system is thought to be largely doseand duration-dependent: whereas relatively low doses of CS have been shown to be effective and relatively safe for long-term therapy of inflammatory diseases, shorter courses of high doses are typically used to treat acute exacerbations of these disorders. ${ }^{4-8}$ With regard to MS, it has been established that high-dose, short-term intravenous CS therapy provides symptomatic relief, improves motor function, and shortens the recovery phase of acute disease-related attacks. ${ }^{9-12}$

\section{Pharmacological properties and mechanisms of action}

Corticosteroids may be administered orally or parenterally. With regard to their pharmacokinetic properties, most CS in clinical use are fairly similar: $\sim 90-95 \%$ of the administered drug is plasma bound, predominantly to corticosteroid-binding globulin and albumin. The plasma half-life is typically between 60 and 90 minutes, whereas the biological half-life often exceeds 24 hours. Secreted by the adrenal glands, these agents are metabolized by the liver.

Corticosteroids have multiple biological effects on numerous organ systems. In this section, we will describe the effects of CS on the immune system, which are thought to be threefold ${ }^{13,14}$ :

1) Very rapid, nonspecific, nongenomic effects occur within seconds after exposure of a cell to high concentrations CS. It appears that these nonspecific, nongenomic effects are the result of a direct interaction of CS with cell membranes. ${ }^{13,14}$

2) Rapid, specific, nongenomic effects of CS are mediated by steroid-selective receptors on the cell surface. These receptors communicate with a second messenger system and occur within a few minutes. ${ }^{15}$ Alternatively, direct physicochemical interactions between CS and the cell membrane could account for these effects. ${ }^{13}$
3) Delayed genomic effects are mediated by cytosolic CS receptors. ${ }^{14}$ Within 30 minutes of a steroid dose, engagement of these receptors leads to the activation of a signaling cascade, and ultimately decreases the transcription of numerous largely proinflammatory mediators. $^{16,17}$

The majority of clinical and paraclinical effects of CS cannot be explained by their very rapid, nonspecific, nongenomic effects, because the therapeutic doses used in most in vivo or in vitro systems would not result in detectable biological activity. Similarly, delayed genomic effects do not correspond with most therapeutic effects of CS. ${ }^{13,18}$ Thus, the rapid, specific nongenomic effects of CS appear to be the most important mechanism by which these agents exert their pharmacological action in the treatment of acute flares of disease. Note also that potencies of CS mediated by the three mechanisms just listed may be inconsistent, and quite variable. It has been shown that the very rapid, nonspecific, nongenomic potencies of commonly used CS differ from their classical potencies mediated by delayed, genomic effects. ${ }^{19}$ This is an important observation, in that the potencies of CS used in clinical practice may need to be reassessed, depending on the pharmacodynamic effects of each specific agent.

As we have already mentioned, CS mediate a multitude of pharmacological effects. One of the potentially more relevant effects concerning MS and other inflammatory disorders of the central nervous system (CNS) involves leukocyte trafficking across biological membranes.

Leukocytes have to transverse the basement membrane (basal lamina) of brain venules during their egress from peripheral blood to access the CNS parenchyma. Matrix metalloproteinases (MMPs) are proteolytic enzymes considered to be one of the major physiologic mediators of cell migration through the extracellular matrix. ${ }^{20}$ In animal studies, intracerebral injection of MMP-2 was shown to disrupt the bloodbrain barrier through the disintegration of the basal lamina around capillaries. In 1996, Rosenberg et al. ${ }^{21}$ demonstrated that compromise of the blood-brain barrier can be alleviated with CS therapy in patients with acute MS exacerbations. Specifically, the authors measured MMPs in the CSF of patients with MS. Patients who received CS to treat an acute episode of MS had CSF sampled before and after 3 days of high-dose CS therapy. The levels of MMP-9 in the CSF decreased significantly and the levels of tissue inhibitors of MMP increased after CS administration. ${ }^{21}$ These observations indicate that increased expression of MMP-9 in the CSF is associated with a compromised blood-brain barrier, and that MMPs are a potential target of CS treatment. 


\section{Rapid, specific nongenomic effects of CS in the CNS} and secondary lymphoid organs

The first membrane-bound CS receptor on neurons was identified in 1991 and has been associated with regulation of CS behavior. ${ }^{22}$ In addition, CS surface receptors and some of their functions have been identified in multiple organ systems, including secondary lymphoid organs. Lysis of lymphoma cells as a result of CS therapy appears to be receptor-mediated. ${ }^{23-25}$ High doses of CS are also proapoptotic, through specific, nongenomic effects that are distinct from cell death by lysis. A cascade of morphological events eventually leads to cell shrinkage, chromatin condensation, and caspase activation. ${ }^{26}$ Ultimately, these changes result in alterations of cellular and mitochondrial membranes, and oligonucleosomal DNA fragmentation. ${ }^{26}$ In contrast to cell lysis observed in cell necrosis, cell death by apoptosis largely preserves the integrity of the dead cell. The cells are eliminated by phagocytosis, and the induction of inflammatory responses against the dead cell is often completely absent. Although molecular and cellular events associated with apoptosis have been reported to generate a reservoir of self-antigens with the potential to initiate inflammatory autoimmune conditions, apoptosis is widely considered integral to the maintenance of a healthy, self-tolerant immune system. ${ }^{27}$

$\mathrm{CS}$ in therapeutic doses significantly reduced the respiration of concavalin-A-activated rat thymocytes and human lymphocytes. ${ }^{28,29}$ In contrast, quiescent cells remained unaffected. ${ }^{28,29}$ Thus, CS clearly possess a very favorable pharmacological profile for the treatment of immune-mediated disorders, in which proliferating leukocytes are one of the main therapeutic target.

\section{Glucocorticosteroids in animal models of MS}

Experimental autoimmune encephalomyelitis (EAE) is the prototypical animal model of MS. ${ }^{30}$ EAE can be induced by immunization with a CNS myelin autoantigen, or through the passive adoptive transfer of myelinspecific, activated, encephalitogenic $\mathrm{CD}^{+}$type $1 \mathrm{~T}$ helper-cell lymphocytes. ${ }^{30}$ Depending on the genetic background of the experimental animal, an acute fulminate, chronic, or relapsing-remitting clinical disease course can be observed. ${ }^{30}$ Pathologically, there is evidence of demyelination, inflammation, and neurodegeneration within the CNS.

Our knowledge of the effects of CS in EAE is very limited. The earliest evidence that CS may have beneficial effects in inflammatory CNS disorders was provided by Levine et al., ${ }^{31}$ who demonstrated that therapeutic adrenalectomy enhanced the clinical signs of EAE. McCombe et al. ${ }^{32}$ showed that a single dose of dexamethasone $(4 \mathrm{mg} / \mathrm{kg})$ at disease onset resulted in a significant reduction in the numbers of $\mathrm{T}$ lymphocytes infiltrating into the spinal cord. Limiting dilution analysis further revealed that the number of autoantigen-specific $\mathrm{T}$ cells was also decreased. ${ }^{32}$ Notably, the numbers of apoptotic cells in the spinal cords of treated animals were increased compared with those observed in animals treated with saline. ${ }^{32}$ This finding was consistent with the work of Pender et al., ${ }^{33}$ who had previously demonstrated that cell apoptosis may be a major physiological mechanism of cell removal in acute EAE brain lesions. In the McCombe et al. ${ }^{32}$ study, however, apoptosis could not entirely explain the reduction in inflammatory infiltrates.

\section{INTERVENTION FOR EXACERBATIONS}

\section{Objectives of acute intervention for MS exacerbations}

That application of anti-inflammatory treatment for MS-related exacerbations serves to accelerate recovery from the attack of functional disability and potentially mitigates the severity of the attack itself is supported by a number of investigations. ${ }^{34-37}$ All of these studies were preceded by a clinical trial of ACTH compared with placebo for treatment MS attacks, a monumental milestone in the management of relapsing forms of the disorder. $^{38}$

With respect to the use of CS and ACTH for treating MS attacks, an evidence-based assessment of these agents was undertaken by the Therapeutics and Technology Assessment Committee of the American Academy of Neurology that considered disease-modifying therapies for MS. ${ }^{3}$ In terms of using CS and ACTH, the Committee concluded as follows:

1) Treatment with CS serves to accelerate recovery from attacks of MS and that it is appropriate to consider using these agents (based on class I and II studies with a type A recommendation).

2) Long-term benefits on the disease course has not been demonstrated (Type B recommendation).

3) Compelling evidence does not currently exist to favor the utilization of a particular type of agent, route of administration, or dosage (although most neurologists use high doses of these agents for the treatment of attacks).

CS and ACTH have the capability of restoring the integrity of the blood-brain barrier, thereby influencing mononuclear trafficking mechanisms. In a similar fashion, these agents can serve to reduce the activity of MMPs, and have potent antiedema effects, which can help prevent neuronal membrane dysfunction. Recent evidence has demonstrated that steroids may enhance the fidelity of electrical transmission in demyelinated axonal segments, as evidenced by augmentation of motor evoked potentials elicited by transcranial magnetic stimulation. $^{39}$ 
TABLE 1. Features of Corticosteroid Regimens for Acute Exacerbations

Mechanisms

Evidence-Based Medicine

Lab Assessments

Treatment Regimens
Immunosuppressive; immunomodulatory; adhesion molecules and metalloproteinases

Class I and II evidence for accelerating recovery from relapses. Some class II evidence for pulse steroid treatment controlling relapses and progression

Acute monitoring for 1) diabetes patients; 2) anticoagulation patients; 3) glaucoma patients (intraocular pressure)

1) intravenous methylprednisolone, $1 \mathrm{~g} /$ day for 3-7 days (for relapses)

2) ACTH (as ACTHAR Gel), 80-120 units/day i.m. or s.c. for either

a) 2-3 weeks or

b) 1 week*

3) Oral prednisone, 500-1250 mg/day, divided, for 3-7 days (for relapses)

4) Smoothie Medrol: $1 \mathrm{~g}$ methylprednisolone mixed in smoothie (fruit pureed with ice cream, juice, yogurt, or milk) or juice taken orally with breakfast for 3-7 days (for relapses)

5) Dexamethasone, 160-200 mg/day p.o. or i.v., divided, for 3-7 days for (relapses)

6) Various tapering regimens

a) Prednisone, $200 \mathrm{mg} /$ day for 4 days, then $100 \mathrm{mg} /$ day for 4 days

b) Methylprednisolone dose pak

c) Dexamethasone, $20 \mathrm{mg} /$ day for 4 days, then $16 \mathrm{mg} /$ day for 4 days

*The lead author's preference is for the 1-week regimen.

One analysis of the benefit of CS or ACTH for MS exacerbations considered only randomized double-blind controlled trials, involving 377 patients across six investigations. ${ }^{40}$ Both agents were found to exhibit beneficial effects when compared with placebo with respect to prevention against disease deterioration at the time of the attack, or providing stabilization of existing symptoms within the first 5 weeks of treatment. Only one of these studies provided information concerning the remote benefits of treatment on future disease activity $(n=51)$ in terms of exacerbations or disease progression. No differences were identified to favor active treatment with methylprednisolone versus placebo at 1 year. ${ }^{41}$

How soon after the onset of symptoms steroid treatment can and should be initiated is related to two separate issues: whether to treat a specific relapse immediately, and how late into a relapse it is effective to treat. The issue of timing depends on the nature of the relapse. In general, the clinician should treat a major relapse immediately but take a wait-and-see approach before deciding to treat minor relapses. Treatment can be successfully initiated as late as 1-2 months into a relapse.

\section{Practical considerations for treating exacerbations}

The general approach to treating acute MS exacerbations has often involved use of intravenous agents such as methylprednisolone (MP) or dexamethasone (TABLE 1 ), and perhaps the most commonly prescribed agent has been intravenous MP $1 \mathrm{~g}$ /day for periods of 3 or more days (generally 3-5; occasionally longer). A point of great controversy has been whether an oral steroid taper is useful following the initial intravenous pulse of steroid. There are no good data to answer this question.

The Optic Neuritis Treatment Trial (ONTT) did show that intravenous MP followed by an oral prednisone taper was associated with superior efficacy in reducing the risk of a second event of inflammatory demyelination, compared with patients randomized to placebo or low-dose oral prednisone alone (without an antecedent intravenous pulse) ${ }^{35}$ From a small optic neuritis study, Herishanu et al. ${ }^{43}$ showed that high-dose intravenous steroids, without a subsequent taper, were associated with a higher risk of recurrent attacks, compared with patients treated with placebo. The Herishanu et al. ${ }^{43}$ study suggests the potential value of a tapering phase of anti-inflammatory treatment following the initial highdose intravenous pulse of treatment. In another study, the use of a steroid taper was associated with short-term reductions in brain volume, compared with those treated without a taper. ${ }^{42}$ Nevertheless, many practitioners do not in fact use tapering schedules after an initial highdose pulse of steroids.

Intravenous CS can be administered either as a single daily dose or as divided daily doses. There is an open issue of which is preferable and more effective. Oncedaily administration saturates steroid carriers and might lead to higher tissue levels, but the details of any difference are unknown. The use of single versus multiple daily infusions is also potentially an issue in terms of insurance coverage. Although existing data are not conclusive, generally administration as a single daily dose is more acceptable to patients, more practical, and more cost effective.

An alternative approach to intravenous MP, one that is evidence-based with respect to accelerating recovery from MS attacks, involves the use of ACTH gel (which can be used parenterally by the intramuscular or subcutaneous route of administration) (TABLE 1). Although the ACTH gel has some theoretical advantages, it is more expensive than steroids, has more side effects, and gives less consistent results. ACTH can be used when patients 
are unresponsive to $\mathrm{CS}$, or in cases in which its positive effects on bone via stimulation of dehydroepiandrosterone and mineralocorticoids may be desirable. This latter feature is of practical importance, in that patients under disease-modifying therapy either intramuscularly or subcutaneously can easily administer ACTH by either route, greatly facilitating independent home treatment by a route of administration that is intimately familiar to the patient. In addition to these potential advantages, ACTH stimulation of endogenous CS agents may represent another benefit over exogenous formulations. Nevertheless, no controlled studies are yet available to corroborate this hypothesis.

Although treatment of exacerbations for those taking interferons or glatiramer acetate can involve both the initial pulse (CS or ACTH) with or without a taper, for patients under natalizumab therapy it may be prudent to use only short courses of these agents (up to 3 days), without a taper-as suggested variously by the U.S. Food and Drug Administration, Biogen Idec (Cambridge, MA), and Elan (Dublin, Ireland) via the Touch program. Further, we have no data to suggest how many such CS courses would be considered safe in patients also taking natalizumab. The premise here is an attempt to avoid intensified compromise of immune surveillance that could be the pretext for progressive multifocal leukoencephalopathy, a virally mediated process (via the JC virus) that has been associated with natalizumab as a part of combination therapy. ${ }^{43-45}$

With respect to the question of using low-dose regimens of CS for the treatment of milder attacks, this most certainly has been and will continue to be prescribed. The problem with advocating this approach is the patently obvious lack of information with respect to efficacy, and indeed evidence that mild attacks benefit from any treatment. The one important and conspicuous exception is the experience derived from the ONTT; in this trial the use of low-dose oral prednisone $(1 \mathrm{mg} / \mathrm{kg})$ was associated with a worse outcome (approximately double the recurrence rate of optic neuritis) than in either the high-dose (intravenous, followed by an oral taper) steroid limb or the placebo group. ${ }^{33}$ Although there are always individual exceptions (comorbid conditions, patient characteristics, features of the attack), high rather than low doses of CS and ACTH should generally be used to treat MS exacerbations in part because there are more data demonstrating efficacy and safety.

An important but highly contentious issue relates to the timing of CS treatment intervention. Although most agree that treatment should proceed as soon an exacerbation is confirmed (and as one worthy of intervention), there are in fact no good data to compare the efficacy of such treatments if there is a delay in starting CS. Thus, even though an exacerbation and its resultant deficits may be ongoing (perhaps as long as a week or more), physicians can still consider using CS to hasten the resolution of the attack.

\section{Orally administered corticosteroids}

A number of studies (albeit generally a small number) have demonstrated that high-dose oral steroid regimens appear comparable to those administered parenterally, with similar benefit and tolerability. ${ }^{34,46-48}$ The advent of oral steroid regimens for treating MS attacks obviates the need for intravenous, intramuscular, or subcutaneous ACTH administrations, with the obvious advantages of markedly reduced cost and greater ease of use. Important investigations have shown that high-dose oral steroid treatment has no impact on gastric permeability changes, emphasizing the principle that steroid-related gastritis is not related to direct effects of these agents on gastric mucosa, but rather is likely secondary to systemic mechanisms (regardless of the route of administration). ${ }^{49}$ Perhaps most noteworthy has been evidence demonstrating similar bioavailability of CS under oral and parental administration..$^{50,51}$

In our anecdotal experience, a number of oral steroid regimens are highly effective, well tolerated, and extremely cost effective (TABLE 1). For example, some of us routinely use a formulation we refer to as smoothiemedrol. The pharmacist dissolves $1 \mathrm{~g}$ of MP powder in $25 \mathrm{~mL}$ of water (dextrose 5\% in water; D5W) and provides this to the patient (three vials for a 3-day course). The patient then mixes the solution in an 8-ounce smoothie (e.g., strawberry-banana) or juice and drinks the concoction with breakfast (sometimes at night, if steroid behavioral activation is shorter in onset). Some patients find the taste of the smoothie-medrol cocktail aversive. As an alternative, we prescribe $150-200 \mathrm{mg}$ of dexamethasone (as a single dose, or divided over breakfast and lunch) mixed in $25 \mathrm{~mL}$ of D5W by the pharmacist, which the patient then adds to an 8-ounce smoothie or fruit juice. This latter formulation is very well tolerated and nearly tasteless (the lead author has been forced to taste both formulations, as brought to clinic by an activist patient).

Others among our group have routinely used oral prednisone tablets, which are well accepted by patients despite the large number of pills to be swallowed at once. Others prescribe oral dexamethasone tablets (TABLE 1). Dexamethasone may have advantages over other formulations, given that is has no mineralocorticoid effects and is therefore associated with fewer overall symptom complaints (other than a higher predilection for behavioral changes), but this has not been shown to be important with short-term pulse therapy.

Ultimately, the oral CS regimens are often the most simple, convenient, and cost effective regimens to employ for the treatment of MS exacerbations. The response to CS for exacerbations is highly variable. As such, some 
of us will offer a second course of therapy if we perceive that the exacerbation continues, or is worsening despite intervention. In these cases, we counsel patients on the importance of having reasonable expectations to be derived from CS treatments. Nonetheless, if patients fail to respond to oral CS (perhaps after a week or so) and exhibit ongoing features of the exacerbation, we will then consider following the oral regimen with an intravenous course of steroids.

An important pearl with respect to the use of CS or ACTH is consideration of the individual patient's past experience and preferences with respect to efficacy, tolerability, and convenience. Patients often have very strong impressions concerning their experiences with steroids, and may ultimately be best able to determine which formulations have been the most effective. This is not surprising, given that the individual agents and their regimens are in fact different, and that pharmacogenetic factors may determine differential efficacy and tolerability across patients. We suggest that providers directly query patients about these experiences and their subjective but nonetheless important observations that may indicate intolerance.

\section{INTERACTIONS AND SIDE EFFECTS}

\section{Drug interactions}

Corticosteroids have traditionally been considered to have relatively few clinically important drug-drug interactions, but interactions with warfarin (Coumadin) can cause either increased or decreased warfarin effect, and interactions between corticosteroids and enzyme-inducing antiepileptic drugs such as phenytoin (Dilantin), phenobarbital, and carbamazepine (Tegretol) are now recognized as common, because CS are also metabolized by the cytochrome P-450 (CYP) isoenzymes. This may result in reduced serum levels of one or both drugs because of enzyme induction, and may require increasing the dose of both drugs. The need for monitoring anticonvulsant levels should be considered, because dexamethasone has clearly been shown to significantly reduce phenytoin serum concentrations and has led to an increase in seizures in MS patients with epilepsy. ${ }^{52}$ Given that little is understood about the best dose of CS for the treatment of relapses, this has not led us to adjust CS dose in patients using these therapies. In contrast, enzyme-inhibiting drugs-notably valproic acid (Epival), fluvoxamine (Luvox), nefazdone (Serzone), calcium channel blockers such as diltiazem (Cardizem) and verapamil, troleandomycin, erythromycin, clarithromycin (Biaxin), and oral antifungal agents-may impair the metabolism of CS and may therefore increase toxicity.

\section{Adverse effects of CS and ACTH}

A number of well-recognized adverse events can be associated with the use of steroids and ACTH:
1) Behavioral effects

a) Depression

b) Euphoria

c) Agitation

d) Anxiety

e) Psychosis (rarely)

2) Sleep derangements

3) Hypertension

4) Diabetes

5) Lipid derangements

6) Gastritis and reflux

7) Edema

8) Skin changes

9) Metallic taste

10) Bone loss

11) Weakness

12) Avascular necrosis (rare with acute use)

13) Cataracts and glaucoma (rare with acute use)

14) Anticoagulation problems for those on warfarin

In most circumstances, this adverse-event profile is associated with the chronic utilization of these agents. Some patients, however, can exhibit highly conspicuous and intolerable side effects even with very short courses of CS and ACTH. Among the more commonly encountered problems are insomnia, emotional lability (depression, irritability, euphoria, and rarely psychosis), dyspepsia, headache, and hypertension. Despite the strong preoccupation with the idea that CS frequently produce gastric irritation, there has been little of an evidence basis for this, particularly in the context of pulse therapy in MS. ${ }^{49}$ Nevertheless, some of us commonly place such patients on either $\mathrm{H} 2$ blockers or proton pump inhibitors. For those with hypertension who require pulse steroid therapy, we have such patients carefully monitor their blood pressure readings at home and report problems to the clinic. Similarly, diabetic patients who are treated with CS need to carefully and consistently monitor blood glucose levels to ensure avoidance of severe hyperglycemia (potentially requiring insulin) and a hyperosmolar state.

Patients with diabetes mellitus and those who are taking warfarin should be monitored during steroid therapy, as should patients at risk for the development of other serious side effects related to hyperglycemia and drug interaction-induced alterations in prothrombin time. Despite these potential side effects, the vast majority of MS patients can be effectively and safely treated with any of the regimens that we describe (TABLE 1).

Patients on CS therapy may exhibit a diminished response to toxoids and live or inactivated vaccines, due to inhibition of antibody response. CS may also potentiate 
the replication of some organisms contained in live attenuated vaccines. If possible, routine administration of vaccines or toxoids should be deferred until CS therapy is discontinued.

An association between use of systemic CS and cleft lip and palate $(\mathrm{OR}=2.59 ; 95 \% \mathrm{CI}=1.18-5.67)$ has been demonstrated in humans, after many animal studies suggested this association. ${ }^{52,53}$ During early pregnancy, when cleft disorders develop, CS should be used only if the potential benefit justifies the potential risk to the fetus.

\section{SAMPLE PATIENT VIGNETTES AND REVIEW}

\section{Vignette 1}

A 19-year-old woman with relapsing-remitting multiple sclerosis (RRMS) presents with new-onset optic neuritis. She is treated with oral methylprednisolone at 1 g/day for 3 days, followed by prednisone taper. She recovers her vision.

\section{Vignette 2}

2. A 24-year-old woman with RRMS currently treated with interferon (IFN) $\beta 1$-a presents to hospital with a severe sensorimotor myelitis. Exam shows hypesthetic paraparesis and a level at T8. Magnetic resonance imaging- shows a lesion within the spinal cord at T6. She admits to being frequently noncompliant with her injections. In fact, she last had a dose of IFN $\beta 1-1$ a a full 3 months ago. IFN-neutralizing antibodies are negative. There is a nationwide shortage of methylprednisolone. Instead, she is treated with intravenous dexamethasone at $160 \mathrm{mg} /$ day for 5 days, followed by an oral prednisone taper (TABLE 1). Physical therapy is started. She improves significantly over 2-3 weeks. Given intensive counseling and education, she promises to be compliant with her IFN $\beta 1-1 \mathrm{a}$ injections.

\section{Pearls}

Currently there are no data to support the following practices:

1) use of a specific steroid for exacerbations, with the exception of a first event of optic neuritis (in which case the standard of care is high-dose intravenous methylprednisolone, $1 \mathrm{~g} /$ day for 3 days, followed by a prednisone taper);

2) use of a specific steroid for relapse reduction;

3) a particular route of administration for steroids; and

4) use of a particular dose of steroid for treatment of relapses.

\section{CONCLUSION AND FUTURE DIRECTIONS}

CS and ACTH are effective and generally well tolerated when carefully and systematically used for treating MS exacerbations. Although there is a broad diversity of potential treatment regimens, neurologists must gain experience with those they feel comfortable in prescribing. Without doubt, we are in great need of more evidencebased studies to determine whether there are in fact true differences among the various agents, doses, and their applications in specific pulse and tapering regimens. Unfortunately, a formidable challenge to executing such studies is the absence of any patent rights on these agents, and the long-held perception that the use of these agents does not alter the ultimate course of the MS disease process.

We do know from the ONTT that short-term courses of steroids applied to patients with optic neuritis do not in fact exert any long-term benefits. Nevertheless, these agents are typically given at the time of an exacerbation for only a defined period before being withdrawn. This approach alone is certainly not a logical therapy for a disease that is multiphasic and protracted in most patients. We also know from the ONTT that those who received high-dose intravenous steroids within 8 days of optic neuritis onset had an $\sim 50 \%$ reduction in the risk of another inflammatory demyelinating event for up to 2 years (despite being treated for only 14 days). ${ }^{35}$ It is conspicuous that continuously administered interferons and glatiramer acetate show only about a one-third reduction in attack rate over 2 years. Future studies should then also consider whether CS and ACTH may provide disease-modifying effects if used on a regular basis. Disease-modifying effects are the subject of the companion article in this issue, the second of this two-part series.

Acknowledgments: This project was supported by Lone Star Chapter of the National Multiple Sclerosis Society funds awarded to the UT Southwestern MS Center and awards to E.M.F. from the following sources: Once Upon A Time Foundation, the Cain/Denius Comprehensive Center for Mobility Research, the Irene Wadel and Robert Atha Fund, the Kenney Marie Dixon Pickens Fund, the Jean Ann and Steve Brock Fund for Medical Sciences, and the Hawn Foundation.

\section{REFERENCES}

1. Uhthoff W. Untersuchungen über die bei der multiplen Herdsklerose vorkommenden Augenstorungen [In German]. Arch Psychiatr Nervenkrankh 1889;20:55.

2. Hansen BS, Hussain RZ, Lovett-Racke AE, Thomas JA, Racke MK. Multiple toll-like receptor agonists act as potent adjuvants in the induction of autoimmunity. J Neuroimmunol 2006;172:94103.

3. Goodin D, Frohman EM, Garmany GP Jr, et al. Disease modifying therapies in multiple sclerosis: report of the Therapeutics and Technology Assessment Subcommittee of the American Academy of Neurology and the MS Council for Clinical Practice Guidelines. Neurology 2002;58:169-178 [Erratum in: Neurology 2002;59: 480]. 
4. Dalakas MC. Current treatment of the inflammatory myopathies. Curr Opin Rheumatol 1994;6:595-601.

5. Kirwan JR; The Arthritis and Rheumatism Council Low-Dose Glucocorticoid Study Group. The effect of glucocorticoids on joint destruction in rheumatoid arthritis. N Engl J Med 1995;333:142_ 146.

6. Hall S, Conn DL. Immunosuppressive therapy for vasculitis. Curr Opin Rheumatol 1995;7:25-29.

7. Guillevin L, Lhote F. Distinguishing polyarteritis nodosa from microscopic polyangiitis and implications for treatment. Curr Opin Rheumatol 1995;7:20-24.

8. Youssef PP, Haynes DR, Triantafillou S, et al. Effects of pulse methylprednisolone on inflammatory mediators in peripheral blood, synovial fluid, and synovial membrane in rheumatoid arthritis. Arthritis Rheum 1997;40:1400-1408.

9. Droogan AG, Crockard AD, McMillan SA, Hawkins SA. Effects of intravenous methylprednisolone therapy on leukocyte and soluble adhesion molecule expression in MS. Neurology 1998;50: 224-229.

10. Filipovic SR, Drulovic J, Stojsavljevic N, Levic Z. The effects of high-dose intravenous methylprednisolone on event-related potentials in patients with multiple sclerosis. J Neurol Sci 1997;152: 147-153.

11. Patzold T, Schwengelbeck M, Ossege LM, Malin JP, Sindern E. Changes of the MS Functional Composite and EDSS during and after treatment of relapses with methylprednisolone in patients with multiple sclerosis. Acta Neurol Scand 2002; 105:164-168.

12. Humm AM, Z'Graggen WJ, Buhler R, Magistris MR, Rosler KM. Quantification of central motor conduction deficits in multiple sclerosis patients before and after treatment of acute exacerbation by methylprednisolone. J Neurol Neurosurg Psychiatry 2006;77: 345-350.

13. Buttgereit F, Wehling M, Burmester GR. A new hypothesis of modular glucocorticoid actions: steroid treatment of rheumatic diseases revisited. Arthritis Rheum 1998;41:761-767.

14. Gold R, Buttgereit F, Toyka KV. Mechanism of action of glucocorticosteroid hormones: possible implications for therapy of neuroimmunological disorders. J Neuroimmunol 2001;117:1-8.

15. Wehling M. Specific, nongenomic actions of steroid hormones Annu Rev Physiol 1997;59:365-393.

16. Boumpas DT, Chrousos GP, Wilder RL, Cupps TR, Balow JE. Glucocorticoid therapy for immune-mediated diseases: basic and clinical correlates. Ann Intern Med 1993;119:1198-1208.

17. Adcock IM, Caramori G, Ito K. New insights into the molecular mechanisms of corticosteroids actions. Curr Drug Targets 2006;7: 649-660.

18. Falkenstein E, Norman AW, Wehling M. Mannheim classification of nongenomically initiated (rapid) steroid action(s). J Clin Endocrinol Metab 2000;85:2072-2075.

19. Buttgereit F, Brand MD, Burmester GR. Equivalent doses and relative drug potencies for non-genomic glucocorticoid effects: a novel glucocorticoid hierarchy. Biochem Pharmacol 1999;58:363368.

20. Yong VW, Power C, Forsyth P, Edwards DR. Metalloproteinases in biology and pathology of the nervous system. Nat Rev Neurosci 2001;2:502-511.

21. Rosenberg GA, Dencoff JE, Correa N Jr, Reiners M, Ford CC. Effect of steroids on CSF matrix metalloproteinases in multiple sclerosis: relation to blood-brain barrier injury. Neurology 1996; 46:1626-1632.

22. Orchinik M, Murray TF, Moore FL. A corticosteroid receptor in neuronal membranes. Science 1991;252:1848-1851.

23. Gametchu B. Glucocorticoid receptor-like antigen in lymphoma cell membranes: correlation to cell lysis. Science 1987;236:456461.

24. Gametchu B, Watson CS, Wu S. Use of receptor antibodies to demonstrate membrane glucocorticoid receptor in cells from human leukemic patients. FASEB J 1993;7:1283-1292.

25. Diba F, Watson CS, Gametchu B. 5'UTR sequences of the glucocorticoid receptor $1 \mathrm{~A}$ transcript encode a peptide associated with translational regulation of the glucocorticoid receptor. J Cell Biochem 2001;81:149-161.
26. Chaudhari BR, Murphy RF, Agrawal DK. Following the TRAIL to apoptosis. Immunol Res 2006;35:249-262.

27. Navratil JS, Liu CC, Ahearn JM. Apoptosis and autoimmunity. Immunol Res 2006;36:3-12.

28. Buttgereit F, Krauss S, Brand MD. Methylprednisolone inhibits uptake of $\mathrm{Ca}^{2+}$ and $\mathrm{Na}^{+}$ions into concanavalin A-stimulated thymocytes. Biochem J 1997;326:329-332.

29. Schmidt J, Gold R, Schonrock L, Zettl UK, Hartung HP, Toyka $\mathrm{KV}$. T-cell apoptosis in situ in experimental autoimmune encephalomyelitis following methylprednisolone pulse therapy. Brain 2000; 123:1431-1441.

30. Zamvil SS, Steinman L. The T lymphocyte in experimental allergic encephalomyelitis. Annu Rev Immunol 1990;8:579-621.

31. Levine S, Wenk EJ, Muldoon TN, Cohen SG. Enhancement of experimental allergic encephalomyelitis by adrenalectomy. Proc Soc Exp Biol Med 1962; 111:383-385.

32. McCombe PA, Nickson I, Tabi Z, Pender MP. Corticosteroid treatment of experimental autoimmune encephalomyelitis in the Lewis rat results in loss of $\mathrm{V}$ beta $8.2+$ and myelin basic proteinreactive cells from the spinal cord, with increased total T-cell apoptosis but reduced apoptosis of V beta $8.2+$ cells. J Neuroimmunol 1996;70:93-101.

33. Pender MP, Nguyen KB, McCombe PA, Kerr JF. Apoptosis in the nervous system in experimental allergic encephalomyelitis. J Neurol Sci 1991;104:81-87.

34. Durelli L, Cocito D, Riccio A, et al. High-dose intravenous meth ylprednisolone in the treatment of multiple sclerosis: clinical-immunologic correlations. Neurology 1986;36:238-243.

35. Beck RW, Cleary PA, Anderson MM, et al. A randomized, controlled trial of corticosteroids in the treatment of acute optic neuritis. N Engl J Med 1992;326:581-588.

36. Sellebjerg F, Frederiksen JL, Nielsen PM, Olesen J. Double-blind, randomized, placebo-controlled study of oral, high-dose methylprednisolone in attacks of MS. Neurology 1998;51:529-534.

37. Brusaferri F, Candelise L. Steroids for multiple sclerosis and optic neuritis: a meta-analysis of randomized controlled clinical trials J Neurol 2000;247:435-442.

38. Rose AS, Kuzma JW, Kurtzke JF, Namerow NS, Sibley WA, Tourtellotte WW. Cooperative study in the evaluation of therapy in multiple sclerosis: ACTH vs. placebo-final report. Neurology 1970;20:1-59.

39. Humm AM, Z'Graggen WJ, Buhler R, Magistris MR, Rosler KM. Quantification of central motor conduction deficits in multiple sclerosis patients before and after treatment of acute exacerbation by methylprednisolone. J Neurol Neurosurg Psychiatry 2006;77: $345-50$.

40. Filippini G, Brusaferri F, Sibley WA, Citterio A, Ciucci G, Midgard R, Candelise L. Corticosteroids or ACTH for acute exacerbations in multiple sclerosis.

41. Ciucci G, Midgard R, Candelise L. Corticosteroids or ACTH for acute exacerbations in multiple sclerosis. Cochrane Database Syst Rev 2000;(4):CD001331.

42. Hoogervorst EL, Polman CH, Barkhof F. Cerebral volume changes in multiple sclerosis patients treated with high-dose intravenous methylprednisolone. Mult Scler 2002;8:415-419.

43. Herishanu YO, Badarna S, Sarov B, Abarbanel JM, Segal S, Bearman JE. A possible harmful late effect of methylprednisolone therapy on a time cluster of optic neuritis. Acta Neurol Scand 1989;80:569-574.

44. Stüve O, Marra CM, Jerome $\mathrm{K}$, et al. Immune surveillance in multiple sclerosis patients treated with natalizumab. Ann Neurol 2006;59:743-747.

45. Stüve O, Marra CM, Jerome K, et al. Altered CD4:CD8 T cells ratios in cerebrospinal fluid of natalizumab-treated multiple sclerosis patients. Arch Neurol 2006;63:1383-1387.

46. Stüve O, Marra CM, Petra CD, et al. The potential risk of progressive multifocal leukoencephalopathy with natalizumab therapy: possible interventions. Arch Neurol 2007;64:169-176.

47. Sellebjerg F, Schaldemose Nielsen H, Fredericksen JL, Olesen J. A randomized, controlled trial of oral high-dose methylprednisolone in acute optic neuritis. Neurology 1999;52:1479-1484.

48. Alam SM, Kyriakides T, Lawden M, Newman PK. Methylpred- 
nisolone in multiple sclerosis: a comparison of oral with intravenous therapy at equivalent high dose. J Neurol Neurosurg Psychiatry 1993;56:1219-1220.

49. Barnes D, Hughes RAC, Morris RW, et al. Randomised trial of oral and intravenous methylprednisolone in acute relapses of multiple sclerosis. Lancet 1997;349:902-906.

50. Metz LM, Sabuda D, Hilsden RJ, Enns R, Meddings JB. Gastric tolerance of high-dose pulse oral prednisone in multiple sclerosis. Neurology 1999;53:2093-2096.
51. Morrow SA, Stoian CA, Dmitrovic J, Chan SC, Metz LM. The bioavailability of IV methylprednisolone and oral prednisone in multiple sclerosis. Neurology 2004;63:1079-1080.

52. Lackner TE. Interaction of dexamethasone with phenytoin. Pharmacotherapy 1991;11:344-347.

53. Pradat P, Robert-Gnansia E, Di Tanna GL, Rosano A, Lisi A, Mastroiacovo P; Contributors to the MADRE database. First trimester exposure to corticosteroids and oral clefts. Birth Defects Res A Clin Mol Terato 2003;67:968-970. 